\title{
Realistic Modeling of Chamber Transport for Heavy-Ion Fusion*
}

\author{
W. M. Sharp, D. P. Grote, D. A. Callahan, M. Tabak, \\ E. Henestroza, S. S. Yu, P. F. Peterson, D. R. Welch, \\ D. V. Rose,
}

This article was submitted to: Particle Accelerator Conference 2003, Portland, Oregon, 5/12/2003 - 5/17/2003

\section{June 4, 2003}

Lawrence

Livermore

National

Laboratory 


\section{DISCLAIMER}

This document was prepared as an account of work sponsored by an agency of the United States Government. Neither the United States Government nor the University of California nor any of their employees, makes any warranty, express or implied, or assumes any legal liability or responsibility for the accuracy, completeness, or usefulness of any information, apparatus, product, or process disclosed, or represents that its use would not infringe privately owned rights. Reference herein to any specific commercial product, process, or service by trade name, trademark, manufacturer, or otherwise, does not necessarily constitute or imply its endorsement, recommendation, or favoring by the United States Government or the University of California. The views and opinions of authors expressed herein do not necessarily state or reflect those of the United States Government or the University of California, and shall not be used for advertising or product endorsement purposes.

This is a preprint of a paper intended for publication in a journal or proceedings. Since changes may be made before publication, this preprint is made available with the understanding that it will not be cited or reproduced without the permission of the author.

This work was performed under the auspices of the United States Department of Energy by the University of California, Lawrence Livermore National Laboratory under contract No. W-7405-Eng-48.

This report has been reproduced directly from the best available copy.

Available electronically at http://www.doc.gov/bridge

Available for a processing fee to U.S. Department of Energy

And its contractors in paper from

U.S. Department of Energy

Office of Scientific and Technical Information

P.O. Box 62

Oak Ridge, TN 37831-0062

Telephone: (865) 576-8401

Facsimile: (865) 576-5728

E-mail: reports@adonis.osti.gov

Available for the sale to the public from

U.S. Department of Commerce

National Technical Information Service

5285 Port Royal Road

Springfield, VA 22161

Telephone: (800) 553-6847

Facsimile: (703) 605-6900

E-mail: orders@ntis.fedworld.gov

Online ordering: http://www.ntis.gov/ordering.htm

OR

Lawrence Livermore National Laboratory

Technical Information Department's Digital Library

http://www.llnl.gov/tid/Library.html 


\title{
REALISTIC MODELING OF CHAMBER TRANSPORT FOR HEAVY-ION FUSION*
}

\author{
W. M. Sharp, D. P. Grote, D. A. Callahan, M. Tabak, LLNL, Livermore, CA 94550, USA \\ E. Henestroza, S. S. Yu, LBNL, Berkeley, CA 94720, USA \\ P. F. Peterson, U C Berkeley, Berkeley, CA 94720, USA \\ D. R. Welch, D. V. Rose, Mission Research Corporation, Abluquerque, NM 87110, USA
}

\section{Abstract}

Transport of intense heavy-ion beams to an inertialfusion target after final focus is simulated here using a realistic computer model. It is found that passing the beam through a rarefied plasma layer before it enters the fusion chamber can largely neutralize the beam space charge and lead to a usable focal spot for a range of ion species and input conditions.

\section{INTRODUCTION}

To achieve high gain, indirect-drive targets for heavyion fusion must be driven by a tailored input-energy profile.[1] This profile is designed to launch a series of four shocks through the capsule that compress and accelerate the fuel on a low adiabat. The pulse shape typically consists of a long ( $25 \mathrm{~ns})$, low-power "foot" that heats the hohlraum interior to about $100 \mathrm{eV}$ and launches the first shock. The power is then ramped to the peak value and held for about $8 \mathrm{~ns}$ as the subsequent three shocks are launched. The hohlraum geometry and the required temperature history of the capsule determine the ion-beam current profile. In addition, distributed-radiator targets $[2,3]$ require different ion kinetic energies in the foot and main pulses to compensate for ion range shortening as the hohlraum is heated, and the beam energy must be deposited in an annular region on each end of the 5-mm-radius cylindrical target to produce the necessary radiation symmetry inside the hohlraum. These target requirements put tight constraints on the number, current, and focal radius of the drive beams.

A point design meeting these constraints has recently been worked out [4]. In this paper, we use the electromagnetic particle-in-cell code LSP [5,6] to investigate the chamber transport of the foot and mainpulse beams of this design. The single-beam simulations here include beam neutralization by plasma injected into the chamber-entry beam line, collisional ionization of the beam and background gas, and photoionization by $\mathrm{X}$ rays from the heated target. The results both demonstrate the viability of the point design and explore the effects of the initial emittance and convergence angle of the beam, the choice of ion species, and the spatial profile of the neutralizing plasma.

\footnotetext{
* This work was performed under the auspices of the U. S. Department of Energy by University of California Lawrence Livermore National Laboratory and Lawrence Berkeley National Laboratory under contracts W-7405-ENG-48 and DE-AC03-76SF00098.
}

\section{RESULTS}

\section{Parameters}

The power profile required by the point-design target is built up using beams with different durations, currents, energies, and arrival times, as sketched in Fig. 1. A total of 120 beams is specified, divided into five classes. The lower-energy foot is built up by stacking three types of beam, and the main pulse is formed from two more types. The large number is needed both to keep the current in each beam manageable and to give each type of beam adequate azimuthal symmetry when deposited in the annuli on the target ends.

The point design specifies beams of singly charged bismuth ions (209 amu). However, the simulations here also model beams of xenon (131 amu) to test the sensitivity of the beam focal radius to ion mass. Since target stopping power is principally a function of ion velocity, the energy of ions is roughly proportional to atomic mass $M$, and beam current increases inversely with $M$. The beam perveance, which is a measure of the importance of space charge in transverse dynamics, increases like $M^{2}$. To have the required stopping power, bismuth main-pulse ions require an energy of $4 \mathrm{GeV}$, while xenon ions must have $2.5 \mathrm{GeV}$. Foot pulse energies are $25 \%$ lower. Due to their higher power, the initial foot pulses and the final 48 main pulses are the most challenging to focus, so the beam parameters used here match those two types. These main-pulse beam currents

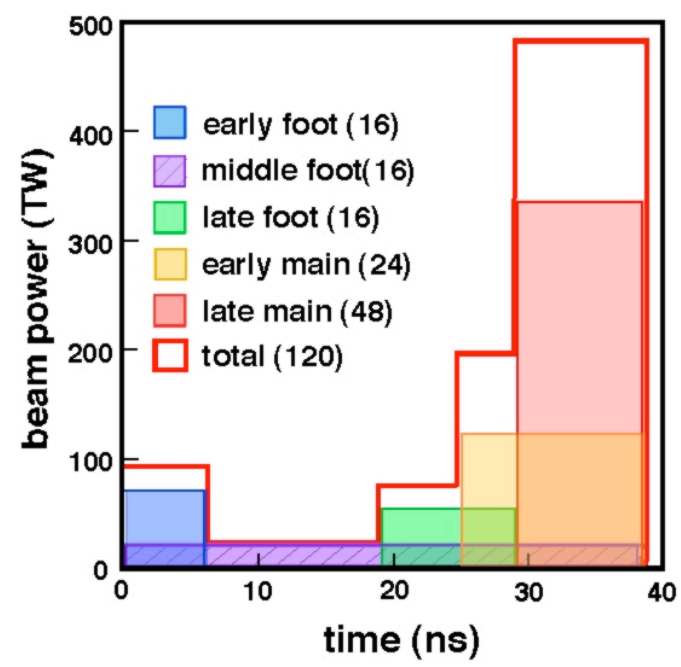

Figure 1: Power deposited on the fusion target by 120 ion beams. Numbers in parentheses give the number of beams of each type. 
are $2 \mathrm{kA}$ for bismuth and $3.2 \mathrm{kA}$ for xenon, and the corresponding foot-pulse currents are $1.5 \mathrm{kA}$ and $2.4 \mathrm{kA}$. In all cases here, beam current falls off in the 3-ns beam ends like a normal ogive, and the radius drops from its mid-pulse value to $2 \mathrm{~cm}$ with a similar S-shaped pattern.

As discussed elsewhere [7] in more detail, each beam is focused on a target location six meters from the last finalfocus magnet. A beam first passes through a conical 3-m pipe lined with a molten-salt vortex to protect the surface. This molten-salt layer is modeled as an insulator with a surface conductivity of $220 \mathrm{~S} / \mathrm{m}$. The beam then enters the 3- $\mathrm{m}$ radius fusion chamber, filled with $\mathrm{BeF}_{2}$ and $\mathrm{LiF}$ vapor from the molten salt jets used to protect the chamber walls. This background gas has a vapor pressure of $7 \mathrm{x}$ $10^{12} \mathrm{~cm}^{-3}$.

Distributed-radiator targets require that at least $90 \%$ of the beam energy be deposited in an annulus on each end of the hohlraum. For the specific target used in the point design, the main pulse must hit an annulus with a halfwidth of $1.8 \mathrm{~mm}$, and the annulus for foot pulses has a 2.2$\mathrm{mm}$ half-width. Therefore, a good criterion for evaluating the simulations is the fraction of energy deposited in a band with a half-width equal to that of the target annulus. This measure ignores the curvature of the annulus but is still a useful approximation.

\section{Effects of Plasma Parameters}

Plasma neutralization after final focus is essential for all the cases discussed here. The maximum perveance of main pulses is $7 \times 10^{-5}$ for bismuth and $1.7 \times 10^{-4}$ for xenon, and that of foot pulses is 2.5 times higher. Analytic work by Olson [8] indicates that the upper perveance limit for ballistic transport is about $1.6 \times 10^{-5}$, so the number of beams would have to be increased roughly ten-fold to meet this condition. Instead, as in Ref. 7, we use a rarefied plasma in the beam entry pipe to neutralize each beam before it enters the chamber. Placing a $10-\mathrm{cm}$ thick layer of plasma with a density of $3 \times 10^{11} \mathrm{~cm}^{-3}$ near each end of the $3-\mathrm{m}$ entry pipe increases beam neutralization from about $30 \%$ near the target to more than $95 \%$ and decreases the rms radius of the beam focal-spot from about $2.5 \mathrm{~cm}$ to less than $2 \mathrm{~mm}$.

While a neutralizing plasma upstream makes a dramatic improvement in beam focus, we find that increasing the plasma density has little effect, so long as there are sufficient plasma electrons in the volume swept out by the beam to fully neutralize the beam space charge. The $3 \mathrm{x}$ $10^{11} \mathrm{~cm}^{-3}$ plasma density used here is about a factor of ten higher than this minimum, but a significant change in the beam focal radius is only seen when the plasma density is lower than about $10^{10} \mathrm{~cm}^{-3}$.

Although the beam focal spot is not sensitive to the plasma density, the axial density profile of the plasma layers is found to have a pronounced effect, particularly that of the first layer encountered by the beam. When the plasma density drops abruptly to zero at the plasma edge, only about $85 \%$ of the energy of a bismuth main pulse falls within the requisite 1.8 - $\mathrm{mm}$ half-width band, but this fraction increases to $92 \%$ when the density falls parabolically over $3-\mathrm{cm}$ and to $97 \%$ for a $6-\mathrm{cm}$ parabolic or normal-ogive edge. An examination of these cases shows that a current of backstreaming electrons nearly equalling the beam current develops as the unneutralized beam approaches the square-profile plasma. This current flows near the beam axis, and the resulting nonlinear space-charge field within the beam causes a substantial emittance increase. Both the electron current and the emittance increase are less for the other plasma profiles. For example, we see only a $10 \%$ emittance increase in the entry pipe for the ogive profile, compared with nearly a doubling for a square plasma edge. For the remaining cases here, we use the more realistic ogival profile.

We have also studied the sensitivity of the beam focal spot to the length plasma. Specifically, we have compared placing a plasma layer near each end of the entry pipe against backfilling the entire entry pipe with plasma. In each case, a 6-cm ogival plasma boundary is used on each plasma edge, and the same $3 \times 10^{11} \mathrm{~cm}^{-3}$ interior density is specified. For both bismuth and xenom main pulses, a plasma backfill increases the energy deposited on the target annulus by about $3 \%$. Nonetheless, we use the layout with two plasma layers for other cases here because it poses fewer engineering problems.

\section{Effect of Initial Emittance}

The robust point design specifies an initial normalized edge emittance of about $2 \mathrm{~mm}$-mrad, allowing less than a factor of five growth from source to target. Simulations show a marked degradation in the beam focal spot when this stringent condition is not met. When the initial emittance of a xenon main pulse is doubled, for example, the energy deposited in a 1.8-mm half-width band drops from $96 \%$ to $90 \%$, and this fraction drops to about $85 \%$ when the emittance is tripled. Bismuth beams a somewhat less sensitive to emittance change, but a tripling of the nominal value still leads to an unusable focal spot. Since the emittance grows only about $20 \%$ in the chamber when a realistic plasma density profile is used, emittance growth in the accelerator is tightly constrained.

\section{Effect of Convergence Angle}

A smaller beam convergence angle is preferable for several reasons. The unshielded solid angle around the target through with neutrons, gamma rays, and debris can escape is proportional to the square of the beam radius, so reducing the convergence angle simplifies shielding. A second benefit is that the beam bundle converging on each end of the hohlraum can have a smaller cone angle, allowing the use of higher-gain targets as well as smaller and less costly final-focus magnets.

The robust point design specifies a maximum convergence angle of 10 -mrad, corresponding to a $6-\mathrm{cm}$ radius after final focus, six meters from the target. LSP simulations of both bismuth and xenon show less than a $5 \%$ drop in the energy deposited within the target annulus when a 7.5-mrad convergence angle is used. This change is substantially less than the $25 \%$ predicted from a simple ballistic-transport model, indicating that residual space 

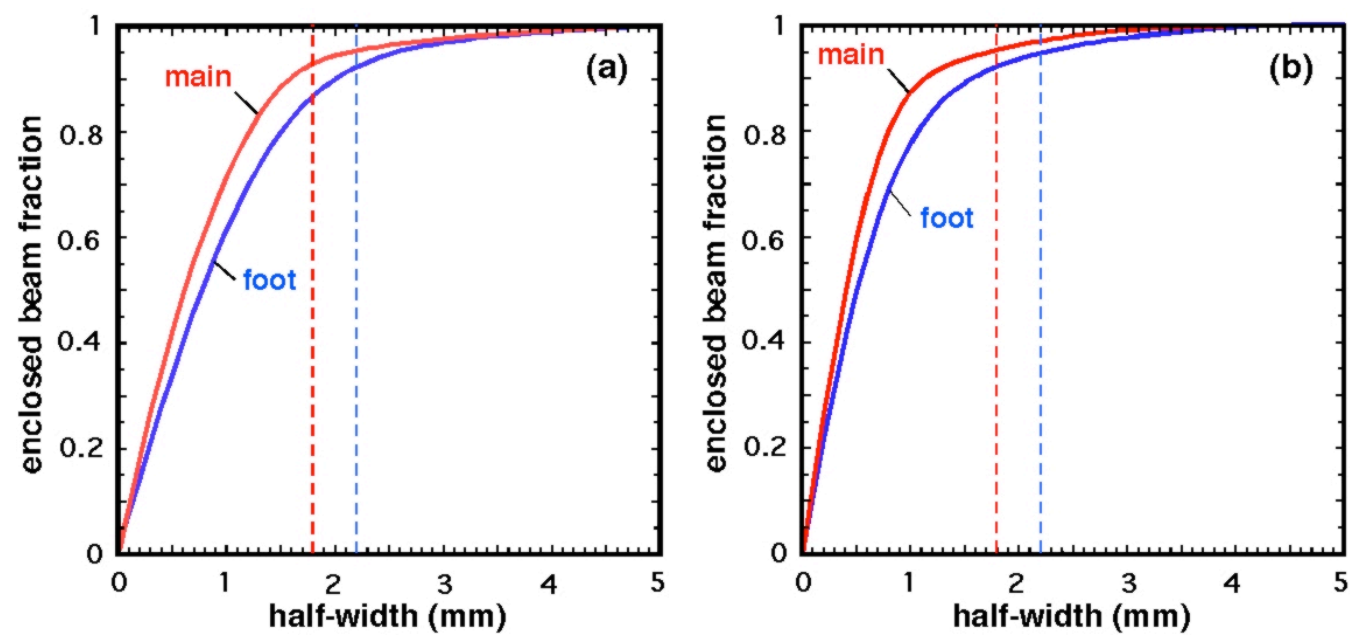

Figure 2: Fraction of beam energy deposited within a band of a specified half-width for (a) xenon and (b) bismuth main and foot pulses. Half-widths of the corresponding target annuli are shown as dashed lines.

charge still plays a role in transverse dynamics. Due to this insensitivity to convergence angle, an optimized design would likely use a smaller value, although the other simulations here use 10-mrad.

\section{Effects of Ion Species}

The choice of beam ion species has a major impact on the cost of a driver. Since the required energy of beam ions is proportional to their atomic mass, and the cost of induction accelerators increases proportionally with the beam energy, power-plant studies favor a low ion mass.

Energy deposition for main and pulses of bismuth and xenon are compared in Fig. 2. While bismuth has better focus near the center of the pulse and is therefore more robust, as expected, both species satisfy the criterion that at least $90 \%$ of beam energy fall within the appropriate target annuli. The main pulse fractions are $96 \%$ for bismuth and $93 \%$ for xenon. Due to the absence of a photoionized plasma, the foot pulses have poorer neutralization near the target, a larger halo, and lower fractional deposition. However, the deposition fractions are only about $1 \%$ less than the corresponding main-pulse values, due to the wider target annulus.

Recent work on beam sources indicates that intense beams of negatively charged halogen ions may be feasible [9]. Such beams could be photoneutralized after final focus by laser and would not need a neutralizing plasma. We have simulated chamber transport of a neutral iodine (127 amu) main pulse, using the same beam power as the point design and including ionization of the beam and background-gas due to collisions and target $X$ rays. Near the target, the net charge of the iodine beam is less than that of a plasma-neutralized xenon beam, and the fraction of energy deposited in a $1.8-\mathrm{mm}$ half-width band exceeds $98 \%$, compared with $93 \%$ for xenon. This improved beam deposition might allow the use of higher emittance beams and permit a simpler chamber design due the absence of plasma neutralization.

\section{CONCLUSIONS}

The chamber-transport simulations here improve on those published with the "robust point design" [4] in several ways. Both the beams and the plasmas used to neutralize them are given more realistic density profiles, boundary conditions in the chamber entry pipe are closer to those expected in a driver, and a much wider range of beam parameters has been investigated. The principal finding of this work is that elements as low in atomic mass as xenon could be used as beam species. Also, both main and foot pulses could have a smaller convergence angle and somewhat higher emittance than previously considered. These changes would permit a more economical driver than is possible using bismuth. Finally, preliminary simulations of initially neutral beams show even better transport characteristics than positive ions.

\section{REFERENCES}

[1] J. Lindl., Phys. Plasmas 2 (1995) 3933.

[2] M. Tabak and D. A. Callahan., Nucl. Instr. and Meth in Phys. Res. A 415 (1998) 75.

[3] D. A. Callahan and M. Tabak, Nucl. Fusion 39 (1999) 1547.

[4] S. S. Yu, et al., "A Robust Point Design for a Heavy Ion Fusion Reactor," to be published Fusion Sci. and Tech. (2003).

[5] T. P. Hughes, et al., Phys. Rev. ST Accel. Beams 2 (1999) 110401.

[6] D. R. Welch, et al., Nucl. Instrum. Meth. Phys. Res A 464 (2001) 134.

[7] W. M. Sharp, et al., "Modeling Chamber Transport for Heavy-Ion Fusion," to be published in Fusion Sci. and Tech. (2003).

[8] C. L. Olson, AIP Conf. Proc. 152 (1986) 215.

[9] L. L. Grisham, "Proof-of-Concept Experiments for Negative Ion Driver Beams for Heavy Ion Fusion," in these proceedings. 